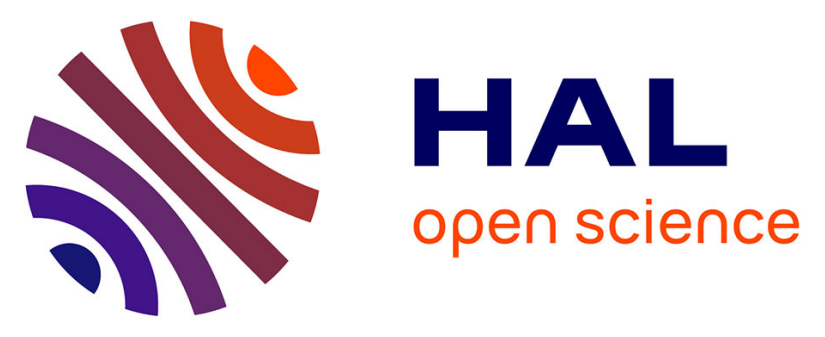

\title{
Estimation of fuel cell operating time for prédictive maintenance strategies
}

\author{
R. Onanena, Latifa Oukhellou, Denis Candusso, Allou Same, Daniel Hissel, \\ Patrice Aknin
}

\section{> To cite this version:}

R. Onanena, Latifa Oukhellou, Denis Candusso, Allou Same, Daniel Hissel, et al.. Estimation of fuel cell operating time for prédictive maintenance strategies. International Journal of Hydrogen Energy, 2010, vol.35 (n.15), pp 8022-9. 10.1016/j.ijhydene.2010.05.039 . hal-00559459

\section{HAL Id: hal-00559459 \\ https://hal.science/hal-00559459}

Submitted on 25 Jan 2011

HAL is a multi-disciplinary open access archive for the deposit and dissemination of scientific research documents, whether they are published or not. The documents may come from teaching and research institutions in France or abroad, or from public or private research centers.
L'archive ouverte pluridisciplinaire HAL, est destinée au dépôt et à la diffusion de documents scientifiques de niveau recherche, publiés ou non, émanant des établissements d'enseignement et de recherche français ou étrangers, des laboratoires publics ou privés. 
Source International Journal of Hydrogen Energy, Vol35,n15, p8022-9

Ed Elsevier, doi:10.1016/j.ijhydene.2010.05.039

The estimation of fuel cell operating time for predictive maintenance strategies

R. Onanena ${ }^{\text {a,b,c }}$, L. Oukhellou ${ }^{\text {c,d }}$, D. Candusso ${ }^{\text {a,c,* }}$, A. Same ${ }^{\text {c }}$, D. Hissel ${ }^{\text {a,b }}$, P. Aknin ${ }^{\text {c }}$

${ }^{a}$ FC LAB, Techn’Hom, rue Thierry Mieg, 90010 Belfort Cedex, France

b FEMTO-ST (UMR CNRS 6174), ENISYS department, University of Franche-Comté, France

c INRETS - LTN, "Le Descartes 2", 2 rue de la butte verte, 93166 Noisy-le-Grand Cedex, France

${ }^{\text {d } C E R T E S ~ U n i v e r s i t e ́ ~ P a r i s ~ 12, ~} 61$ avenue du Gal. de Gaulle, 94100 Créteil, France

* Corresponding author.

Tel.: +33 3845836 33; $\quad$ Fax: +33 $\quad$ I 84583636

E-mail addresses:

raissa.onanena@inrets.fr (Raïssa Onanena),

latifa.oukhellou@inrets.fr (Latifa Oukhellou),

allou.same@inrets.fr (Allou Same),

patrice.aknin@inrets.fr (Patrice Aknin),

denis.candusso@inrets.fr (Denis Candusso),

daniel.hissel@univ-fcomte.fr (Daniel Hissel). 


\begin{abstract}
One of the limiting factors for the spreading of the fuel cell technology is the durability and researches to extend their lifetime are being done world-widely. We present here a pattern recognition approach aiming to estimate fuel cell operating time based on electrochemical impedance spectroscopy measurements. It consists in first extracting features from the impedance spectrum. For that purpose, two approaches have been investigated. In the first one, particular points of the spectrum are empirically extracted as features. In the second approach, a parametric modelling is performed to extract features from both the real and the imaginary parts of the impedance spectrum. In particular, a latent regression model is used to automatically split the spectrum into several segments that are approximated by polynomials. The number of segments is adjusted taking account the $a$ priori knowledge about the physical behaviour of fuel cell components. Then, a linear regression model using different subsets of extracted features is employed for the estimation of fuel cell operating time. The performances of the proposed approach are evaluated on experimental data set to show its feasibility. Being able to estimate the fuel cell operating time, and consequently its remaining duration life, these results could lead to interesting perspectives for predictive maintenance policy of fuel cells.
\end{abstract}

\title{
Keywords:
}

Fuel cell; Durability; Reliability; Diagnostic; Predictive maintenance 


\section{Introduction}

Clean and renewable energy as well as limited greenhouse gas emissions are important issues for producers and consumers of energy. Different ways to deliver cleaner power are investigated worldwide. One of them is to use Fuel Cells (FCs) that appear to be a promising technology and environmentally friendly energy conversion solution for the future, especially for transport applications.

However, the economical viability of FC systems, especially in the transportation sector, depends notably on improving the stack durability and reliability. Indeed, the stack is prone to material degradation (e.g. poisoning of the catalyst sites, loss of proton conductivity in the membrane, corrosion of plates, etc.) and the performance decay induced is strongly linked to the operating conditions (i.e. pollutants in the reactants, insufficient amounts of reactive gas flows versus the load current demand, operating temperature, mechanical constraints on the membrane electrode assemblies, etc.) [1-5]. Typical life requirements range from 5000 hours at least for car applications to 20000 operating hours for bus applications. Moreover, when dealing with durability and reliability, the efficient diagnosis of FC stack and system appears as a major issue. The development of diagnostic schemes can help evaluating the FC state-ofhealth, and thus speed up the development cycle of new technologies (e.g. FC vehicles). Various diagnosis approaches for FC stacks and systems have been developed. They include model-based methods [6-10], gray or black box model approaches using fuzzy logic [11], design of experiment methods [12], neural networks [13], or non-parametric identification by Markov parameters [14]. Recent FC stack diagnosis approaches based on fuzzy clustering [15] and bayesian networks have been proposed [16]. 
This paper presents a pattern recognition based diagnosis aiming to estimate the FC operating time from Electrochemical Impedance Spectroscopy (EIS) measurements done at approximately regular time intervals throughout two different Proton Exchange Membrane (PEM) FC durability tests (conducted on two stacks, noted here as FC1 and FC2). Our analysis uses data collected on FC1 and FC2 to ensure the robustness of the obtained results. Furthermore, with the proposed approach, we are also able to estimate the remaining FC lifetime. For predictive maintenance purposes, it is obviously important to know how FC performances evolve throughout its working.

Fig. 1 - FC operating time estimation on the basis of a pattern recognition based approach.

The complete diagnosis system consists of several steps, shown in Fig. 1. From each recorded impedance spectrum (high dimensional data), a feature extraction is performed to generate features (low dimensional data). The goal of extraction and selection is to find a small number of features among the original ones that are particularly informative for the problem to be solved. Furthermore, dimensionality reduction is also essential when the available training data set is small as it is in our case, otherwise the well known phenomenon of curse of dimensionality could inevitably appear, leading to over-fitting $[17,18]$. Finally, linear regression between a meaningful subset of features and the considered output (operating time) is achieved.

The paper is organised as follows. Section 2 describes the two ageing tests and highlights the link between FC ageing and EIS measurements. Section 3 focuses on the two different feature extraction methods that have been used. Section 4 presents the different solutions for 
operating time estimation based on linear regression and compares the different results obtained with the two feature extraction approaches. Experimental results are reported in this section. Section 5 concludes the paper with some perspectives.

\section{Durability issues}

\subsection{Ageing experiments}

In order to evaluate the influence of different factors on the ageing of FCs, experiments were carried out in FC LAB. The durability tests were performed on two identical small power PEMFC three-cell stacks of about $100 \mathrm{~W}$ during 1000 hours. The testing conditions varied from one stack to another. The first stack (FC1) was operated in nominal and stationary conditions. The load current was constant and equal to $50 \mathrm{~A}$. In the second test, the fuel cell (FC2), was operated under dynamical load current based on a real transportation mission profile (maximum current of $70 \mathrm{~A}$ was reached for an average of $12.5 \mathrm{~A}$ ). Details on the test conditions and kind of current solicitation can be found in [19,20]. During these ageing tests, the stacks were characterised regularly (twice per week). To determine the number of characterisation sequences, a trade-off was found between two different needs: on the one hand, a sufficient amount of collected data for the monitoring of the FC state-of-health, and on the other hand, the minimisation of the perturbations due to the characterisation sequences in the achievement of the durability tests. Except for the two first EIS spectra recorded on FC1 (at the beginning of the ageing test) and for the three first spectra measured on FC2 (at the beginning of the durability test), the characterisations were made at average time intervals of 63 hours for FC1 and 75 hours for FC2. These regular characterisations consist in 
recording polarisation curves and impedance spectra. They have resulted in the constitution of a database made of 17 and 12 impedance spectra for FC1 and FC2 respectively. Note that the complete characterisation test results are reported in $[19,20]$. In this paper, the ageing process will be analysed with the information extracted from impedance spectra only.

\subsection{Electrochemical Impedance Spectrum}

Since the polarisation curve gives information about the static behaviour of the FC, Electrochemical Impedance Spectroscopy (EIS) is used in order to obtain relevant information about its dynamical behaviour. Considering this technique, the FC is placed on its standard (static) operating point and then additional small amplitude solicitation signals are applied. Each recorded impedance spectrum is a series of impedance measurements at discrete frequency points. EIS can be performed in two modes: potentiostatic (voltage control mode) and, like in our case, galvanostatic (current control mode). EIS allows the characterisation of dynamic processes occurring at different timescales in the system. EIS has been widely employed by FC experimenters to study various phenomena, such as diffusion phenomena at cathode side, the ionic conductivity of membranes, pollutant effects and catalyst loading [7,9,21]. EIS can also be used to show the influence of the occurrence of a fault or the evolution of the dynamic behaviour during an ageing process.

\subsection{Effects of FC ageing on impedance spectrum}

During the two ageing tests, the dynamical FC behaviour is investigated using the same characterisation protocol: considering the stationary operating point of $35 \mathrm{~A}$ and a small sinusoidal alternating part around it (with amplitude of $1 \mathrm{~A}$ and frequency range from $10 \mathrm{mHz}$ 
to $30 \mathrm{kHz}$ ). The different ageing test conditions applied on both stacks have led to different EIS results for each FC [19, 20]. As an example, the impedance plots measured during the ageing test conducted on FC1 stack are presented in Fig. 2. We can observe that the FC impedance can be divided into three parts, each part corresponding to a distinct dynamic behaviour:

- a first capacitive $\operatorname{arc}(f<130 \mathrm{~Hz})$,

- $\quad$ a second capacitive arc $(130 \mathrm{~Hz}<f<4 \mathrm{kHz})$,

- $\quad$ an inductive part which is present in high frequencies $(4 \mathrm{kHz}<f)$.

These three parts correspond to physical processes in the FC. At low frequencies, the dynamic impedance is firstly close to the static one (pure resistance corresponding to the slope of the polarisation curve) and then it becomes capacitive due to mass and water transports. At medium frequencies, the charges (electrons and protons) transfer processes become more important than the diffusion phenomena and the second capacitive arc follows the first one. At high frequencies, the inductive behaviour of all connections (internal and external ones) becomes the major influence factor $[19,20]$. The delimiting frequency values presented above are approximate values, and they depend on the FC and its ageing state. Our goal is to use these characteristics, especially their evolution to estimate the FC operating time. Indeed, they seem to be good indicators of the ageing phenomenon in FCs [20].

Fig. 2 - Presentation of hyperparameters in the impedance spectrum (left) and evolution of the impedance spectra for FC1 stack from the initial state (Time : 0) to the final state (Time : 995 hours) (right). 


\section{Feature extraction and selection}

The goal of this processing phase is to generate a subset of features that will represent in the best way the data. Indeed, the spectrum data dimension (the number of its points) is about 50 (50 values of the frequency) whereas the number of observations during its ageing is 29. It is therefore highly recommended to reduce the dimension of the input space, summarise efficiently the measurements, and limit the curse of dimensionality. The underlying motivation is that the data live in a subspace whose intrinsic dimensionality is lower than the one of the original data space [22].

We chose to extract features using two different methods. The first one, presented on the following subsection, consists in the empirical extraction of particular hyperparameters from the spectrum. These hyperparameters contain relevant information regarding the ageing process of FCs. In the second method, the impedance spectrum is separated into two curves that represent the evolution of the real and imaginary parts of the impedance as functions of the frequency. A parameterisation procedure of each of the curves is then performed to generate features.

\subsection{Hyperparameters extraction}

The approach for feature selection is a method based on expert knowledge. The idea is to use as features some particular points of the impedance spectrum referred as hyperparameters that give relevant information on the ageing process. Previous studies presented in $[19,20]$ have focused on identifying characteristic points in the impedance spectrum that can be employed to diagnose FC stacks. We chose the following parameters as features: 
- the polarisation resistance value,

- $\quad$ the minimal value of the imaginary part in the impedance spectrum, its corresponding real part values and its occurring frequency (3 hyperparameters in total),

- the internal resistance value and its corresponding frequency of occurrence (2 hyperparameters).

In Fig. 2, the clear evolution of these parameters during the ageing process can be observed. For example, the minimal value of the imaginary part decreases throughout the experiments. A more detailed analysis of the temporal evolutions of these hyperparameters can be found in $[19,20]$.

\subsection{Feature extraction on the real and imaginary parts of the spectrum}

In this section, we work with the real and imaginary parts of the Nyquist diagram. This enables us to use explicitly the additional information in the frequency variable distributed along the curve. The real and imaginary parts are presented in Fig. 3. It can be seen that an inherent link can be established between the FC ageing phenomenon and the evolution of these curves. Moreover, like on the EIS spectra, three parts representing the three physical processes can also be observed in the imaginary parts of the spectra. Therefore, it seems interesting to extract features from these plots that can be used afterwards to estimate the ageing time of the FC stack.

Fig. 3 - Evolution of the real and imaginary parts of the impedance spectrum versus frequency for FC1 from the initial state (Time : 0) to the final state (Time : 995 hours). 


\subsubsection{Feature extraction on the real part of the impedance}

The real part of the impedance as a function can be approximated by an external model of four parameters related to an extended "logsig” function and defined as follows:

$\operatorname{Re}(\log (f))=\frac{a_{1}}{1+e^{-a_{2}\left(\log (f)-a_{3}\right)}}+a_{4}$

The four model coefficients are determined by minimising a cost function based on a mean square error with the help of simplex method [23]. The behaviour of this model is presented in

Fig. 4. This "global" model does not fit perfectly the experimental data in the low frequencies part (see Fig. 4). However, it still gives good results: the mean error is equal to $1.18 \%$ in the low-frequency range, $1.15 \%$ in the medium frequencies and $1.45 \%$ in high frequencies. A more complex model could give a better approximation of the curves. However, it could also model the noise, and over-fit the curve (i.e. the fitted curve would pass exactly through each experimental point, but would oscillate widely between the experimental points and give a poor representation of the real part). With the proposed model, a trade-off between complexity and accuracy has been considered.

Fig. 4 - Example of real part of the impedance spectrum and its approximation by the external logsig model.

\subsubsection{Feature extraction on the imaginary part of the impedance}


The imaginary part of the spectrum is more informative than the real one. Rather than using a global fitting of this curve, the idea that we investigate here is to partition it into different segments that can be approximated individually by a polynomial. As indicated previously in section 2.3., both number and location of the segments have to be chosen in relation with the physical behaviour of the FC stack.

\section{- Latent regression model}

The feature extraction used here consists of a specific regression model incorporating a discrete hidden logistic process $[24,25]$. This method is a probabilistic-model-based approach to feature extraction. As it will be demonstrated further on, this model is adapted for measurements including smooth or abrupt transitions between regimes.

Let $x=\left(x_{1}, \ldots, x_{n}\right)$ be the $n$ points of the imaginary part of an impedance spectrum where $x_{i}$ is observed for the frequency value $f_{i}$. In the following, $f_{i}$ will denote the logarithm of the frequency $\left(f_{i} \sim \log f_{i}\right.$.) that is more convenient for spectrum representation. This specific regression model assumes that the measurement incorporates $K$ polynomial regimes (or class) and that the switching from one regime to another is controlled automatically by a latent discrete variable $z_{i}$. This latent variable takes its values in the set $\{1, \ldots, K\}$, and represents the class label of the polynomial regression mode. Thus, the observation is assumed to be generated by the following regression model:

$\forall i=1 \ldots n, \quad x_{i}=\beta_{z_{i}}^{T} r_{i}+\sigma_{z_{i}} \varepsilon_{i}$,

where:

$\beta_{z i}{ }^{T}$ is the $(p+1)$-dimensional coefficients vectors of the p-degree polynomial,

$r_{i}=\left(1, f_{i}, \ldots,\left(f_{i}\right)^{p}\right)^{T}$ is the-frequency-dependent $(p+1)$-dimensional vector associated to $\beta_{z i}$, 
$\varepsilon_{i}$ are the independent random variables distributed according to a Gaussian distribution with a zero mean and a unit variance,

and $\sigma_{z i}$ is the variance coefficient associated with $\varepsilon_{i}$. The vector $\varepsilon=\left(\varepsilon_{1}, \ldots, \varepsilon_{n}\right)^{T}$ is therefore the noise vector.

The sequence of the latent variables $z=\left(z_{1}, \ldots, z_{n}\right)$ is a logistic process. It allows the switching from one regression model to another in $K$ models. This process assumes that the variables $z_{i}$, given the frequencies $\left(f_{1}, \ldots, f_{n}\right)$, are generated independently according to the multinomial (or multivariate) distribution $M\left(1, \pi_{i 1}(w), \ldots, \pi_{i K}(w)\right)$, where:

$\pi_{i k}(w)=p\left(z_{i}=k ; w\right)=\frac{\exp \left(w_{k 0}+w_{k 1} f_{i}\right)}{\sum_{j=1}^{K} \exp \left(w_{j 0}+w_{j 1} f_{i}\right)}$,

is the logistic transformation of a linear function of the frequency $f_{i}$ and $w=\left(w_{10}, w_{11, \ldots,} w_{k 0}, w_{k 1, \ldots}, w_{K 0}, w_{K 1}\right)^{T}$ is the parameter vector of the logistic process. The relevance of the logistic transformation in terms of flexibility of transition has been well detailed in [24].

\section{- Parameter estimation}

From the model given by Eq. (2), it can be proven that the variable $x_{i}$ is distributed according to the normal mixture density [26]:

$p\left(x_{i} ; \theta\right)=\sum_{k=1}^{K} \pi_{i k}(w) \phi\left(x_{i} ; \beta_{k}^{T} r_{i}, \sigma_{k}^{2}\right)$ 
where $\Phi\left(. ; \mu, \sigma^{2}\right)$ denotes a monodimensional normal density with mean $\mu$ and variance $\sigma^{2}$, and $\theta$ the parameter vector to be estimated:

$\theta=\left(w, \beta_{1}, \ldots, \beta_{K}, \sigma^{2}, \ldots, \sigma_{K}^{2}\right)$

Assuming that, given $\left(f_{1}, \ldots, f_{n}\right)$, the $x_{i}(i=1, \ldots, n)$ are independent, and that each $x_{i}$ is distributed according to the mixture density defined by Eq. (4), the log-likelihood of $\theta$ can be written as:

$$
\begin{aligned}
& L\left(\theta ; x_{1}, \ldots, x_{n}\right)=\log \prod_{i=1}^{n} p\left(x_{i} ; \theta\right) \\
& =\sum_{i=1}^{n} \log \sum_{k=1}^{K} \pi_{i k}(w) \phi\left(x_{i} ; \beta_{k}^{T} r_{i}, \sigma_{k}^{2}\right)
\end{aligned}
$$

This likelihood cannot be directly maximised, so we use a dedicated Expectation Maximisation (EM) algorithm [26,27] to perform the maximisation. The parameters of the hidden logistic process, in the inner loop of the EM algorithm, are estimated using a multiclass Iterative Re-weighted Least-Squares (IRLS) algorithm [28].

\section{- Measurements approximation}

In addition to perform feature extraction, this regression model can be used to approximate and segment the impedance spectrum. In fact, the approximated measurement at sample $i$ is given by the expectation:

$$
E\left(x_{i} ; \hat{\theta}\right)=\int_{-\infty}^{+\infty} x_{i} p\left(x_{i} ; \hat{\theta}\right) d x_{i}=\sum_{k=1}^{K} \pi_{i k}(\hat{w}) \hat{\beta}_{k}^{T} r_{i},
$$


where $\hat{\theta}$ is the parameter vector obtained at the convergence of the EM algorithm. Thus, since this expectation is a sum of polynomials weighted by the logistic probabilities, it is adapted for signals approximation with both smooth and abrupt transitions.

To perform the feature extraction, since the impedance spectrums include three regimes corresponding to three physical behaviours of the stack (i.e. mass transport, charge transfer, and inductive part), the number of regressive components $K$ is then set to 3 . The degree $p$ of the polynomial regression is set to 3 too, which is adapted to the different regimes in these spectrums. Then the number of features representing the imaginary part of the impedance is equal to 14 (12 polynomial coefficients and the regime transitions' frequencies $f_{1}$ and $f_{2}$ ). Figure 5 illustrates the behaviour of the hidden process regression. The three domains are clearly identified.

Fig. 5 - Original signal, the three polynomials (top), and their corresponding logistic probabilities (bottom) for the parameterisation of EIS imaginary part.

\subsection{Feature selection and estimation of the duration time}

The aim of feature selection is to choose a subset of relevant features from the previously extracted ones that are more informative on the FC ageing and simultaneously the most independent from the "inevitable" model noise. The criterion chosen to assess the relevancy of the feature extraction is the performance of the regression model estimating the operating time when it is trained on the given set of features. Because the number of extracted features is relatively small, an exhaustive search is carried out. Considering a set of features, an 
exhaustive feature selection require evaluating all possible subsets of features by training their associate regression model: This means that all possible feature subsets are considered as inputs of the regression model: each feature is individually evaluated, then subsets of two features, three features, four features and so on, until all the possible subsets are covered. At the end, the selected subset of features is the one that leads to the best performance in terms of operating time estimation, i.e. that results in the smallest mean error in the test set.

The final step of this estimation phase is the evaluation of the performances of the linear regression model. A cross validation is used [18,22]. Because the size of the data is small from a statistical point of view, a particular cross-validation technique, known as the "leaveone-out" technique is carried out as follows. Consider the data made up of $N$ elements. The model is trained using $(N-1)$ elements (the training set), and tested on the $N^{\text {th }}$ element (the test set). The process is repeated until each of the $N$ elements is used as a test set. The error rate is then estimated by computing the average of the $N$ resulting test errors. This computation of the error rate provides an estimation of how well the regression will perform on a new data. In the case of a linear regression, the mean square error has been considered as the error rate. The "leave-one-out" technique is generally expensive in time computation but it can be used in our case because the data set is very limited (29 elements only).

\section{Experimental results and discussion}

With the following experiments, we want to illustrate the capability of the proposed approach to estimate the operating time using the features extracted from the spectrum. The data on which the approach is tested is a dataset made up of 29 impedance spectrum measurements 
carried out on two FCs (FC1 and FC2 as previously described in Section 2.1) during 1000 hours.

\section{1. $\quad$ Results using hyperparameters}

The extraction of parameters using hyperparameters gives six parameters:

- the internal resistance $\left(h_{1}\right)$,

- the polarisation resistance $\left(h_{2}\right)$,

- the minimal value of the imaginary part of the spectrum $\left(h_{3}\right)$ and its corresponding real part $\left(h_{4}\right)$,

- the respective frequencies corresponding to the internal resistance $\left(h_{5}\right)$, and the peak of the spectrum $\left(h_{6}\right)$.

The selection of a subset of relevant features among the initial ones is achieved by an exhaustive search. All the possible parameter combinations are evaluated in terms of mean square error of the operating time. The chosen combination is the one that leads to the minimal rate of the test error. At the end, only the polarisation resistance $\left(h_{1}\right)$ and the imaginary part of highest peak $\left(h_{3}\right)$ are selected. That gives us a set of two features $\left(h_{1}, h_{3}\right)$ for a number of 29 observations.

Fig. 6 - Operating time estimation over the training (left) and the test (right) sets for the linear regression using a subset of hyperparameters. 
Figure 6 illustrates the distribution of mean square error (MSE) over all the operating times among the database. The left part corresponds to the training phase and the right part the test phase. With such a model, the operating time of the FC can be estimated with a mean error of 214 hours over the total duration of 1000 hours (i.e. $21.4 \%$ ).

\subsection{Results using feature extraction on the real and imaginary parts of the impedance spectrum}

The feature extraction methods are applied on each spectrum measurement. These lead to extract 4 features $\left\{a_{i},\right\}_{1 \leq i \leq 4}$ from the real part and 14 features $\left\{\beta_{i, j}, f_{1}, f_{2}\right\}_{1 \leq i, j \leq 4}$ from the imaginary part (3 hidden logistic process regressions with 12 coefficients of the three-order polynomial fitting of the curve and the 2 frequencies delimiting the central polynomial). Therefore, a total set of 18 descriptors are available for each impedance spectrum measurement. Like in section 4.1, a linear regression has been used for the estimation of the operating time.

Considering the low number of observations, the first results obtained with the complete set of features (\#18) with a cross-validation procedure lead to very bad estimation. The curse of dimensionality is clearly reached. Even more than what was the case with the previous feature extraction method, a preliminary feature selection is highly recommended.

The selection of a subset of relevant features among the initial ones is also achieved by an exhaustive search. This has resulted in the selection of the following set of features: $\left\{\beta_{21}, \beta_{23}\right.$, $\left.\beta_{24}, a_{1}, a_{2}, a_{3}, a_{4}\right\}$. The performances are evaluated with the "leave-one-out" technique. The results are presented in Table 1, which illustrates the distribution of mean square error (MSE) over all the operating times among the database. The left part corresponds to the training phase and the right part the test phase. With such a model, the operating time of the FC can be 
estimated with a mean error of 142 hours over the total duration of 1000 hours (i.e. $14.2 \%$ ). This can be explained by the fact that this method describes more accurately the impedance spectrum curve, and that probably important information is kept during the feature extraction.

\subsection{Results using a combination of the two feature extraction approaches}

Figure 7 presents the performances of the regression model that uses a subset of features selected among the whole set of 24 features. Likewise, an exhaustive search has been used to select this subset and a "leave-one-out" cross-validation procedure to evaluate the performances. The mean error is equal to 95 hours over the total duration of 1000 hours (i.e. $9.5 \%)$

Fig. 7 - Operating time estimation over the training (left) and the test (right) sets for the linear regression using a subset of hyperparameters and features extracted from parametric models of real and imaginary parts of the impedance spectra.

Table1 presents the results obtained on the whole data set for the two different approaches.

Table 1 - Linear regression model for the estimation of FCs ageing time using different input descriptors. 


\section{Conclusion}

A method to estimate the operating time of FCs, using a pattern-recognition-based approach on EIS measurements, has been presented in this article. It involves two methods of feature extraction from the impedance spectrum, a feature selection procedure to keep only relevant descriptors and a regression model. While the first feature extraction approach uses hyperparameters extracted from the impedance spectrum, the second approach consists first in a parameterisation of the real part with an external model fitting, and then in the parameterisation of the imaginary part with a specific regression model incorporating a discrete hidden logistic process. Because of the small size of the available data set, a particular attention has been paid to the feature extraction and selection steps.

Evaluations on real data set have shown that FC operating time (respectively lifetime) can be estimated with a mean error of 214 hours over a global operating duration of 1000 hours, when one uses the first feature extraction method, whereas the mean error equals 142 hours when using the second method and falls to 95 hours, when using features extracted using both methods. These are encouraging results, particularly in predictive maintenance context. Improvements can be achieved either by the use of additional features extracted from another characterisation measurement (polarisation curve) and/or more exhaustive dataset. 


\section{References}

[1] S. Zhang, X. Yuan, H. Wang, W. Mérida, H. Zhu, J. Shen, S. Wu, J. Zhang, A review of accelerated stress tests of MEA durability in PEM fuel cells, Int. J. Hydrogen Energy 34(1) (2009), pp. 388-404.

[2] G. Tian, S. Wasterlain, D. Candusso, F. Harel, D. Hissel, X. François, Identification of failed cells inside PEMFC stacks in two cases: Anode/cathode crossover and anode/cooling compartment leak, Int. J. Hydrogen Energy, Article in Press, Corrected Proof, Available online 30 May 2009.

[3] R. Lin, B. Li, Y.P. Hou, J.M. Ma, Investigation of dynamic driving cycle effect on performance degradation and micro-structure change of PEM fuel cell, Int. J. Hydrogen Energy 34(5) (2009), pp. 2369-2376.

[4] M. Hinaje, D. Nguyen, S. Raël, B. Davat, C. Bonnet, F. Lapicque, Impact of defective single cell on the operation of polymer electrolyte membrane fuel cell stack, Int. J. Hydrogen Energy 34(15) (2009), pp. 6364-6370.

[5] J. O'Rourke, M. Ramani, M. Arcak, In situ detection of anode flooding of a PEM fuel cell, Int. J. Hydrogen Energy 34(16) (2009), pp. 6765-6770.

[6] T. Escobet, D. Feroldi, S. de Lira, V. Puig, J. Quevedo, J. Riera, M. Serra, Modelbased fault diagnosis in PEM fuel cell systems, J. Power Sources 192 (1) (2009), pp. 216-223. [7] X. Yuan, H. Wang, J. Colin Sun, J. Zhang, AC impedance technique in PEM fuel cell diagnosis - A review, Int. J. Hydrogen Energy 32(17) (2007), pp. 4365-4380.

[8] N. Fouquet, C. Doulet, C. Nouillant, G. Dauphin-Tanguy, B. Ould-Bouamama, Model based PEM fuel cell state-of-health monitoring via ac impedance measurements, J. Power Sources 159 (2) (2006), pp. 905-913. 
[9] M. A. Rubio, A. Urquia, S. Dormido, Diagnosis of performance degradation phenomena in PEM fuel cells, Int. J. Hydrogen Energy, In Press, Corrected Proof, Available online 5 May 2009.

[10] A. Hernandez, D. Hissel, R. Outbib, Modeling and fault diagnosis of a Polymer Electrolyte Fuel Cell using electrical equivalent analysis, IEEE Trans. on Energy Conversion 25 (1), (2010), pp. 148-160.

[11] D. Hissel, M-C. Péra, J-M. Kauffmann, Diagnosis of automotive fuel cell power generators, J. Power Sources 128 (2) (2004), pp.239-246.

[12] B. Wahdame, D. Candusso, X. François, F. Harel, J-M. Kauffmann, G. Coquery, Design of experiment techniques for fuel cell characterisation and development, Int. J. Hydrogen Energy 34 (2)(2009), pp. 967-980.

[13] C. Nitsche, S. Schroedl, W. Weiss, E. Pucher, Rapid (practical) methodology for creation of fuel cell systems models with scalable complexity, J. Power Sources 145, (2) (2005), pp. 383-391.

[14] Y. Tsujioku, M. Iwase, S. Hatakeyama, Analysis and modelling of a direct methanol fuel cell for failure diagnosis, IEEE IECON Conf., Busan, Korea, 2004.

[15] D. Hissel, D. Candusso, F. Harel, Fuzzy Clustering Durability Diagnosis of Polymer Electrolyte Fuel-Cells dedicated to transportation applications, IEEE Trans. on Vehicular Technology 56 (2007), pp.2414-2420.

[16] L. A. M. Riascos, M. G. Simoes, P. E. Miyagi, A Bayesian network fault diagnosis system for proton membrane exchange fuel cells, J. Power Sources 165 (1) (2007), pp. 267278.

[17] R. E. Bellman, Adaptive Control Processes: A Guided Tour. Princeton University Press, NJ, 1961. 
[18] R. O. Duda, P. E. Hart, D. G. Stork, Pattern classification (2nd ed.), John Wiley and Sons, 2001.

[19] F. Harel, X. Francois, D. Candusso, M-C. Pera, D. Hissel, J-M. Kauffmann, PEMFC durability test under specific dynamic current solicitation linked to a vehicle road cycle, Fuel Cells 7 (2) (2007), pp. 142-152.

[20] B. Wahdame, D. Candusso, X. François, F. Harel, M-C. Péra, D. Hissel, J-M. Kauffmann, Comparison between two PEM fuel cell durability tests performed at constant current and under_solicitations linked to transport mission profile, Int. J. Hydrogen Energy 32 (17), (2007), pp. 4523-4536.

[21] X. Yan, M. Hou, L. Sun, D. Liang, Q. Shen, H. Xu, P. Ming, B. Yi, AC impedance characteristics of a $2 \mathrm{~kW}$ PEM fuel cell stack under different operating conditions and load changes, Int. J. Hydrogen Energy 32 (17) (2007), pp. 4358-4364.

[22] C. M. Bishop, Pattern recognition and machine learning, Springer, 2006.

[23] J. A. Nedler, R. Mead, A simplex method for function minimization, Computer Journal 7 (1965), pp. 308-313.

[24] F. Chamroukhi, A. Samé, G. Govaert, P. Aknin, Time series modeling by a regression approach based on a latent process, Neural Networks 22, (5-6) (2009), pp. 593-602.

[25] R. Onanena, F. Chamroukhi, L. Oukhellou, D. Candusso, P. Aknin, D. Hissel, Estimation of fuel cell life time using latent variables in regression context, 8th International Conference on Machine Learning and Applications (ICMLA), Miami, 2009.

[26] G. J. McLachlan, D. Peel, Finite mixture models, Wiley series in probability and statistics, New York, 2000.

[27] A. P. Dempster, N. M. Laird, D. B. Rubin, Maximum likelihood for incomplete data via the EM algorithm, Journal of the Royal Statistical Society, Series B, 39 (1977), pp.1-38. 
[28] P. Green, Iteratively Reweighted Least Squares for Maximum Likelihood Estimation, and some robust and resistant alternatives, Journal of the Royal Statistical Society, Series B, 46 (1984), pp.149-192. 


\section{Figure captions}

Fig. 1 - FC operating time estimation on the basis of a pattern recognition based approach.

Fig. 2 - Presentation of hyperparameters in the impedance spectrum (left) and evolution of the impedance spectra for FC1 stack from the initial state (Time : 0) to the final state (Time : 995 hours) (right).

Fig. 3 - Evolution of the real and imaginary parts of the impedance spectrum versus frequency for FC1 from the initial state (Time : 0) to the final state (Time : 995 hours).

Fig. 4 - Example of real part of the impedance spectrum and its approximation by the external logsig model.

Fig. 5 - Original signal, the three polynomials (top), and their corresponding logistic probabilities (bottom) for the parameterisation of EIS imaginary part.

Fig. 6 - Operating time estimation over the training (left) and the test (right) sets for the linear regression using a subset of hyperparameters.

Fig. 7 - Operating time estimation over the training (left) and the test (right) sets for the linear regression using a subset of hyperparameters and features extracted from parametric models of real and imaginary parts of the impedance spectra. 


\section{Table captions}

Table 1 - Linear regression model for the estimation of FCs ageing time using different input descriptors. 